\title{
Modeling the mechanical properties of lattice structures made by selective laser melting
} \author{
A. V. Soklakov ${ }^{2}$, D. S. Mikhaluk' ${ }^{2}$ A. A. Popovich ${ }^{1}$ \\ ${ }^{\dagger}$ maria.chukovenkova@multiphysics.ru
}

V.Sh. Sufiiarov ${ }^{1}$, A. V. Orlov ${ }^{1}$, E. V. Borisov ${ }^{1}$, V. V. Sokolova ${ }^{1}$, M. O. Chukovenkova ${ }^{\dagger, 2}$,

\begin{abstract}
${ }^{1}$ Peter the Great Saint Petersburg Polytechnic University, 29 Polytechnicheskaya St., St. Petersburg, 195251, Russia ${ }^{2} J S C$ "Center of Engineering Physics Simulation and Analysis", 15 Kondratiyevskiy Av., bld. 2, St. Petersburg, 195197, Russia

Nowadays, materials with graded density are of great interest. These materials are manufactured with the use of additive technologies and consist of multiple lattice structures with variable parameters and topology. By varying the parameters of the lattice structures, one can adjust the mechanical properties of the material as required. The paper proposes an original methodology for predictive modeling of mechanical properties of graded materials with the help of numerical simulation tools for the further design of the human femur endoprosthesis. The developed methodology is based on varying the topology of lattice structures with the consideration of the technological capabilities of additive manufacturing. The materials under the study allow creating endoprostheses with mechanical properties close to those of the human femur. The periodic structure of these materials ensures ingrowing of bone tissue into the endoprosthesis. Endoprostheses made of materials with graded density are superior to the traditionally manufactured prostheses. Endoprostheses manufactured by means of traditional methods are of higher stiffness than bones, thus, during exploitation they can cause bone damage or fracture. The authors applied a combination of biological and mechanical selection criteria for various zones of endoprosthesis and defined variants of topologies of the lattice structures corresponding to the mechanical properties and geometry of the human femur. With the use of the selected quasi-optimal lattice structures topologies, a femur-like endoprosthesis prototype can be designed for its further fabrication with the use of additive manufacturing methods.
\end{abstract}

Keywords: additive manufacturing, property modeling, finite element methods, lattice structures, Ti-6Al-4V.

УДК: 539.3, 621.762

\section{Моделирование механических свойств ячеистых структур, изготовленных селективным лазерным плавлением}

\author{
Суфияров В.Ш. ${ }^{1}$, Орлов А. В. ${ }^{1}$, Борисов Е. В. ${ }^{1}$, Соколова В. В. ${ }^{1}$, Чуковенкова М. О. ${ }^{\dagger}$, \\ Соклаков А. В. ${ }^{2}$, Михалюк Д. С. ${ }^{2}$, Попович А. А. ${ }^{1}$ \\ ${ }^{1}$ Санкт-Петербургский политехнический университет Петра Великого, ул. Политехническая, 29, \\ С.-Петербург, 195251, Россия \\ ${ }^{2} \mathrm{AO}$ «Центр инженерно-физических расчетов и анализа», пр-т. Кондратьевский, 15, корп. 2, \\ С.-Петербург, 195197, Россия
}

В настоящее время большой интерес представляют материалы с градиентной плотностью. Подобные материалы изготавливаются с применением аддитивных технологий и представляют собой множество ячеистых структур, имеющих различную конфигурацию и топологию. Управляя параметрами ячеистых структур, возможно получать требуемые механические характеристики материала. В статье предложена оригинальная методика моделирования механических свойств подобных материалов с помощью инструментов численного моделирования, для возможности дальнейшего проектирования эндопротеза бедренной кости человека. Методика, разработанная авторами, основана на варьировании топологии ячеистых структур материала с учетом технологических возможностей аддитивного производства. Исследуемые в работе материалы позволяют создавать эндопротезы, механические 
свойства которых приближены к механическим свойствам бедренной кости, а их пористая структура, в свою очередь, способствует врастанию живой ткани вглубь протеза. Протезы, изготовленные из материалов с градиентной плотностью, имеют большое преимущество перед протезами, изготовленными традиционными методами. Эндопротезы, изготовленные традиционными методами, имеют высокую жесткость по сравнению с жесткостью кости, что может повлечь за собой разрушение кости в процессе эксплуатации или стать причиной перелома. Авторами применена комбинация биологических и механических критериев выбора топологии ячеистых структур и определены варианты ячеистых структур, которые соответствуют по механическим и геометрическим свойствам бедренной кости человека. С использованием отобранных квази-оптимальных топологий ячеистых структур можно перейти к проектированию прототипа бедренного эндопротеза с механическими свойствами, приближенными к свойствам бедренной кости для последующего изготовления методами аддитивного производства.

Ключевые слова: аддитивные технологии, моделирование свойств, метод конечных элементов, ячеистые структуры, ВТ6.

\section{1. Введение}

Использование аддитивных технологий позволяет создавать материалы с градиентной плотностью. Подобные материалы представляют собой множество ячеек с переменными параметрами. Варьируя конфигурацией ячеек можно управлять механическими характеристиками изготавливаемого изделия. Квазипериодическая структура таких материалов может применяться для изготовления, например, эндопротезов, а возможность управлять параметрами ячеистых структур позволяет получить изделие, механические свойства которого приближены к свойствам кости. Пористая структура, в свою очередь, обеспечивает врастание костной ткани в протез [1-3].

Подбор параметров ячеистых структур можно проводить с помощью математического моделирования [4]. В работе [5] с использованием программного пакета ABAQUS авторы осуществляют расчет модуля упругости материала в зависимости от конфигурации пористых ячеек. Модуль упругости варьировался путем подбора толщины отдельных прутков, из которых состоит ячейка, а также размера этих ячеек, таким образом, чтобы он соответствовал модулю упругости костной ткани при его использовании в качестве имплантата.

В работе [6] рассматривается возможность использования конечно-элементного анализа с использованием коммерческих программных пакетов для моделирования свойств ячеистых структур, изготовленных с помощью аддитивных технологий. Компьютерные модели ячеистых образцов с различными параметрами отдельных ячеек сгенерированы авторами работы в программном пакете MATLAB, а расчеты образцов из ячеистых структур и их комбинаций проведены с использованием программного пакета ANSYS.

Цель данной работы - с помощью инструментов численного моделирования определить варианты топологий ячеистых структур, которые соответствуют по механическим и геометрическим свойствам бедренной кости человека, для последующего изготовления методами аддитивного производства эндопротеза бедренного сустава с механическими характеристиками, максимально приближенными к реальному биологическому объекту. Критериями выбора топологий ячеек являются: объемная плотность, эффективный модуль упругости, размер пор и предел прочности.

\section{2. Постановка задачи}

Для моделирования в работе использовался пакет конечно-элементного анализа ANSYS 19.1. Подготовка геометрических моделей ячеистых структур произведена в CAD-модуле ANSYS SpaceClaim. Построение конечноэлементной сетки выполнено при помощи ANSYS Meshing. Постановка задачи, выполнение расчетов и обработка полученных результатов проведены в модуле ANSYS Mechanical.

Авторами рассмотрено 6 вариантов конфигураций ячеек с различным расположением прутков, заключенных в единичный объем - куб, при фиксированных габаритных размерах куба, и постоянных толщинах прутка в рамках одной ячейки (Рис. 1). Параметры ячейки - $2.5 \times 2.5 \times 2.5$ мм, при толщинах прутка, равных 0.3, 0.4, 0.6, 0.8, 1 мм. Зависимость эффективных механических свойств ячеек в случае выбора значений толщин прутков между рассмотренными принимается линейной. Система названий конфигураций ячеек определена авторами в соответствии с направлениями присутствующих в ячейке прутков: $\mathrm{N}$ - прутки направлены по диагоналям; R - центральный вертикальный пруток; Со - прутки, расположенные на четырех вертикальных ребрах ячейки; $\mathrm{Cr}$ - прутки, проходящие по диагоналям вертикальных граней ячейки, $\mathrm{M}$ - прутки проходят из центра ячейки к серединам горизонтальных рёбер. Конфигурации рассматриваемых структур образованы сочетанием различных вариантов направлений прутков при постоянной образующей конфигурации N.

В бедренной кости человека выделяется два характерных участка, имеющих различную структуру, a, следовательно, и различные механические свойства: кортикальная (компактная) и трабекулярная (губчатая) кость (Рис. 2) [7,8].

Для того, чтобы изготовить протез, имеющий механические характеристики, приближенные к механическим характеристикам кости, необходимо определить

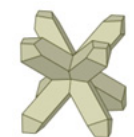

$\mathrm{N}$

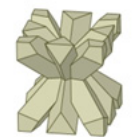

$\mathrm{MN}$

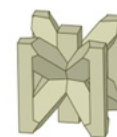

CoRN

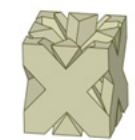

CrMN

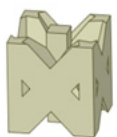

CoCrRN

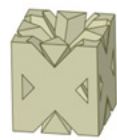

CoCrRMN

Рис. 1. Конфигурации ячеек.

Fig. 1. Configurations of cells. 


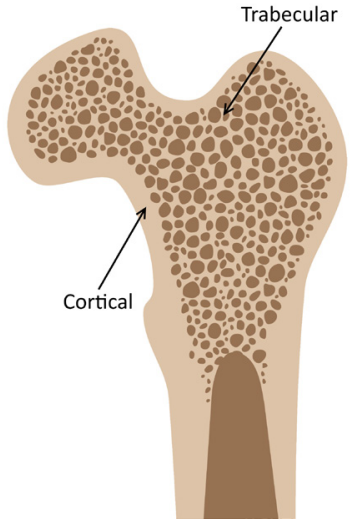

Рис. 2. Участки бедренной кости человека.

Fig. 2. Bone regions in human femur.

подходящие ячеистые структуры, эффективные свойства которых будут соответствовать механическим свойствам кости. Это позволит обеспечить прочность кости в сочленении с эндопротезом [9,10]. Выбор подходящих конфигураций и топологий является основной целью настоящей работы. Авторами выделено три основных параметра, по которым производился отбор ячеек: модуль упругости, размер пор, предел прочности. Для исследования авторами выбрано шесть представительных вариантов конфигураций ячеек: $\mathrm{N}$, $\mathrm{MN}, \mathrm{CoRN}, \mathrm{CrMN}, \mathrm{CoCrRN}$ и CoCrRMN с различным коэффициентом объемного заполнения (Рис. S1, дополнительный материал).

Эффективные механические свойства ячеек определялись с помощью численного моделирования эксперимента на сжатие для единичной ячейки и образца из ячеистой структуры. Исследуется широкий диапазон кривой нагружения в упругой и пластической зонах, вплоть до достижения предела прочности ячеистой структуры.

Модель пластичности, использованная для моделирования одноосного сжатия - мультилинейная. Для мультилинейной модели пластичности исходными данными являются: модуль Юнга E, коэффициент Пуассона $\mu$ и зависимость напряжения $\sigma$ от относительной пластической деформации $\varepsilon$. Учет пластического деформирования при моделировании механических свойств обусловлен стремлением учесть реальную модель материла прутков и получить эффективные механические свойства ячеек в широком диапазоне. Материал, используемый для производства ячеек - титановый сплав ВТ6. Титановый сплав широко применяется в производстве протезов из-за его хорошей биосовместимости, высокой износостойкости и коррозионной стойкости [11]. Механические свойства сплава ВТ6 определены путем обработки результатов проведенного авторами эксперимента на растяжение образцов круглого сечения, изготовленных селективным лазерным плавлением и последующей термической обработкой [12-15]. Механические свойства ВТ6 приведены в Табл. 1 и 2.

Для решения задачи методом конечных элементов решается уравнение $[K]\{u\}=\{F\}$, где $[K]-$ глобальная матрица жесткости, $\{u\}-$ вектор узловых перемещений, $\{F\}-$ вектор внешних сил.

Поскольку рассматривается поведение образцов за пределом текучести, то применяется нелинейная модель упруго-пластического материала, представляющая собой мультилинейную диаграмму, связывающую компоненты девиаторов напряжений и пластических деформаций с применением изотропного упрочнения. Для численного решения на каждом шаге применяется метод Ньютона-Рафсона [16]. Этот метод представляет собой итерационную процедуру решения нелинейных систем уравнений.

Конечно-элементная модель для задачи одноосного сжатия элементарной ячейки показана на Рис. 3. Размер конечного элемента в расчетной модели принят равным 0.2 мм, количество узлов 5434 .

\section{3. Отбор ячеек по размеру пор}

Размер пор для кортикальной части кости соответствует диапазону $0.4-0.8$ мм, для трабекулярной $-0.8-1.2$ мм [17-18]. Крупные поры (0.8-1.2 мм) выполняют в протезе функцию дренажа (обеспечивают подачу органического вещества вглубь эндопротеза), малые (0.4-0.8 мм)

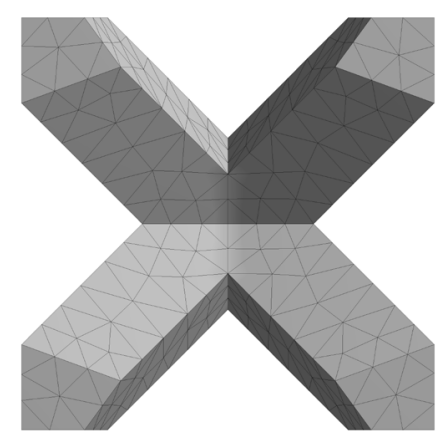

Рис. 3. Конечно-элементная модель элементарной ячейки. Fig. 3. Finite element model of the unit cell.

Табл. 1. Параметры линейно-упругой модели материала ВТ6. Table 1. Linear elastic material model parameters of the Ti-6Al-4V material.

\begin{tabular}{|c|c|}
\hline $\begin{array}{c}\text { Модуль Юнга, МПа } \\
\text { Young's modulus, МРa }\end{array}$ & 113232 \\
\hline $\begin{array}{c}\text { Коэффициент Пуассона } \\
\text { Poisson's ratio }\end{array}$ & 0.36 \\
\hline
\end{tabular}

Табл. 2. Характеристики материала ВТ6 для мультилинейной пластической модели.

Table 2. Ti-6Al-4V characteristics for a multilinear plastic material model.

\begin{tabular}{|c|c|c|c|c|c|c|c|}
\hline $\begin{array}{c}\varepsilon, \mathrm{MM} / \mathrm{MM} \\
\varepsilon, \mathrm{mm} / \mathrm{mm}\end{array}$ & 0 & 0.00096 & 0.00349 & 0.00620 & 0.011729 & 0.02025 & 0.06296 \\
\hline $\begin{array}{c}\sigma, \mathrm{M \Pi a} \\
\sigma, \mathrm{MPa}\end{array}$ & 684.5 & 915.1 & 970.4 & 1006.8 & 1054.3 & 1096.1 & 1177.6 \\
\hline
\end{tabular}


служат для срастания протеза с костными тканями [3]. На данном этапе исследования необходимо провести выбор топологии и размера прутка, для которых размеры пор попадают в установленные условия.

Размер пор в исследуемых моделях определяется размером вписанной сферы в ячейку, такой подход уже использовался ранее [19]. Установлено, что для некоторых конфигураций существует два различных по размерам типа вписанных сфер. (Рис. S2, дополнительный материал).

Далее было проведено исследование изменения размера пор в ячейках в зависимости от толщины прутков для различных конфигураций, для каждой ячейки найдено по две поры (Рис. S3, дополнительный материал).

На основе полученных результатов выбраны ячейки и толщины прутков, для которых размеры пор попадают в установленные диапазоны: $\mathrm{N}$ при толщине прутков 0.8 и 1 мм; CoRN при толщине прутков 0.8 и 1 мм; MN при толщине прутков 0.6, 0.8 и 1 мм; CoCrRN при толщине прутков 0.4 и 0.6 мм; CrMN при толщине прутков $0.4,0.6$ и 0.8 мм; CoCrRMN при толщине прутков $0.3,0.4$ и 0.6 мм.

\section{4. Отбор ячеек по модулю упругости}

Значение эффективного модуля упругости кортикальной части кости находится в диапазоне 14-28 ГПа, модуля упругости трабекулярной части 0.1-4 ГПа [20-22].

Для определения эффективного модуля упругости ячеек для каждого варианта топологии поставлена задача одноосного сжатия для элементарной ячейки. На нижних гранях ячейки действует условие ограничения перемещения по нормали без трения. На верхних гранях приложено малое перемещение (Рис. S4, дополнительный материал).

Эффективный модуль упругости определен по формуле $E_{\mathrm{ef}}=$ ForceReaction $/ S \times \varepsilon$, где Force Reaction значения реакции в опоре, возникающей в результате действия перемещения, $S-$ площадь ячейки, $\varepsilon-$ относительная деформация.

Проведенные вычисления эффективного модуля упругости ячеек с разными толщинами прутков позволили установить зависимости, показанные на Рис. 4. Удовлетворяющие условиям модуля упругости оказались следующие ячейки: $\mathrm{CrMN}$ с толщиной прутка $\sim 0.3-0.34$ мм для трабекулярной части кости, $\mathrm{CoCrRN}$ с толщиной прутка $\sim 0.43-0.55$ мм, MN с толщиной прутка $\sim 0.65-0.68$ мм, CoCrRMN с толщиной прутка $\sim 0.4-0.48$ мм (Рис. 3).

\section{5. Отбор ячеек по пределу прочности}

Значения диапазона эффективного предела прочности для трабекулярной части соответствует диапазону 10-130 МПа, для кортикальной 100-200 МПа [17-18]. На данном этапе исследования необходимо провести выбор конфигурации и размера прутка, для которых предел прочности попадает в установленные диапазоны.
Для определения эффективного предела прочности исследуемых конфигураций ячеек поставлена задача, моделирующая эксперимент на сжатие образца из ячеистой структуры. Во избежание краевых эффектов при моделировании эксперимента используется не одна ячейка, а образец $3 \times 3 \times 3$. Нижняя грань модели образца ограничена в перемещениях по всем направлениям, на верхней грани задано конечное перемещение 5 мм (Рис. S5, дополнительный материал).

Результатом расчета является зависимость силы, возникающей в опоре, от перемещения. Значение эффективного предела прочности $\left(\sigma_{\text {ult }}\right)$ определяется по формуле: $\sigma_{\text {ult }}=F_{\text {ult }} / S_{\text {specimen }}$. Значением силы $F_{\text {ult' }}$, соответствующей предельной (разрушающей) нагрузке, принимается максимальное значение усилия, возникающего в опоре поперечным сечением $S_{\text {specimen }}$.

Значение предела прочности определено для всех вариантов топологий с различными толщинами прутков. Сравнение типовой расчетной зависимости нагрузки от перемещения с экспериментальной для образца из ячеистых структур с топологией $\mathrm{N}$ при толщине прутка 0.8 мм приведено на Рис. 5.

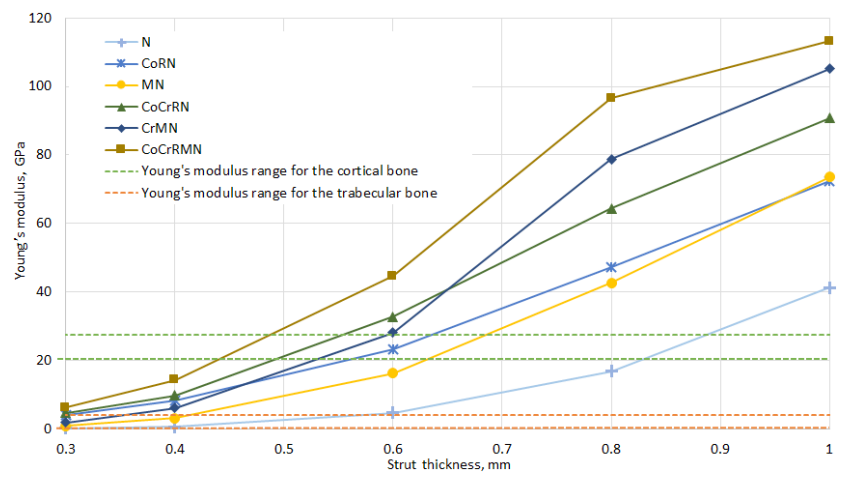

Puc. 4. (Color online) Зависимость эффективного модуля упругости ячеек от толщины прутка.

Fig. 4. (Color online) Dependence of the effective Young's modulus of cells on the strut thickness.

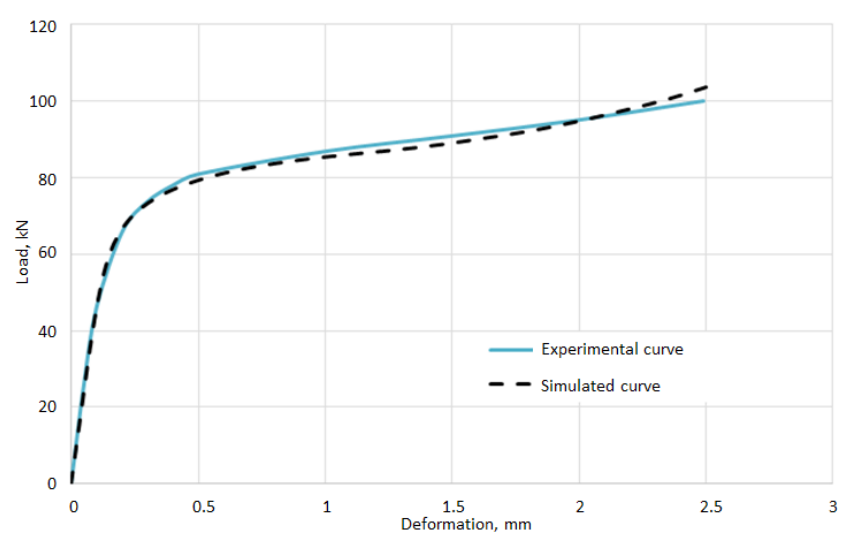

Pис. 5. Зависимость усилия от перемещения для образца из ячеистой структуры с конфигурацией $\mathrm{N}$ при толщине прутка 0.8 мм.

Fig. 5. Stress-displacement relation for a lattice structure specimen with topology $\mathrm{N}$ and a strut thickness of $0.8 \mathrm{~mm}$. 


\section{6. Результат отбора ячеек}

Обобщенные результаты моделирования для отобранных вариантов ячеек показаны на Рис. 6, который отражает зависимость эффективного предела прочности ячейки от её модуля упругости. Для каждого варианта конфигурации ячейки приведены размеры пор (выноски на точках графика на Рис. 5).

На графике также отмечены области пределов прочности трабекулярной и кортикальной кости.

Из результатов расчета видно, что по требованиям соответствия механических свойств ячеек механическим свойствам трабекулярной кости, проходят ячейки следующих конфигураций и топологий: $\mathrm{N}$ с толщиной прутка $\sim 0.48-0.56$ мм, $\mathrm{MN}$ с толщиной прутка $\sim 0.3-0.42$ мм, CrMN с толщиной прутка $\sim 0.3-0.35$ мм.

Для кортикальной кости не найдено ячеек, соответствующих по пределу прочности. Для решения этой проблемы принято решение рассмотреть комбинации ячеек c подходящим по значению эффективным модулем упругости и размером пор. Подходящими ячейками оказались CoCrRN при толщине прутков $0.4,0.45,0.5,0.55$ мм и CoCrRMN при толщине прутков $0.4,0.48$ и 0.5 мм. Для дальнейшего исследования получены образцы $2 \times 2$, состоящие из двух ячеек различных топологий, располагающихся в шахматном порядке (Рис. 7). Из приведенных выше конфигураций ячеек составлено семь комбинаций, проведено исследование на эффективный предел прочности и модуль упругости.

На Рис. 8 представлена зависимость эффективного предела прочности образцов от модуля упругости.

Из результатов расчета определены три комбинации ячеек, эффективные свойства которых соответствуют кортикальной кости не только по модулю упругости и размеру пор, но и удовлетворяют требованиям по пределу прочности: CoCrRMN с толщиной прутка 0.4 мм и CoCrRN с толщиной прутка 0.45 мм; CoCrRN с толщиной прутка 0.4 мм и CoCrRMN с толщиной прутка 0.45 мм; CoCrRN с толщиной прутка 0.4 мм и CoCrRMN с толщиной прутка 0.48 мм.

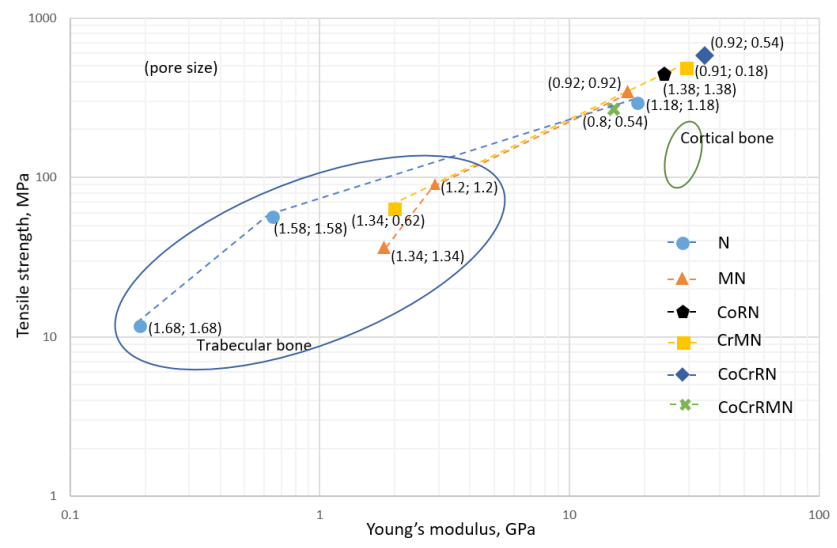

Pис. 6. (Color online) Зависимость эффективного предела прочности ячеек от их модуля упругости.

Fig. 6. (Color online) Dependence of the effective tensile strength of cells on Young's modulus.

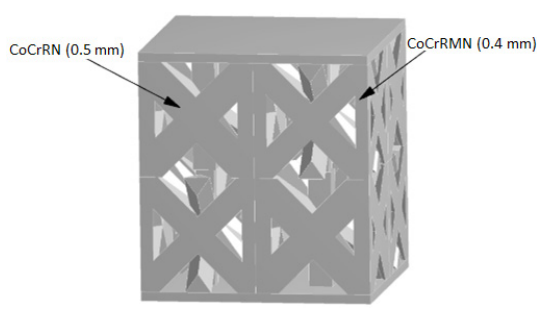

Рис. 7. Образец из комбинации ячеистых структур. Fig. 7. Specimen of combined cells.

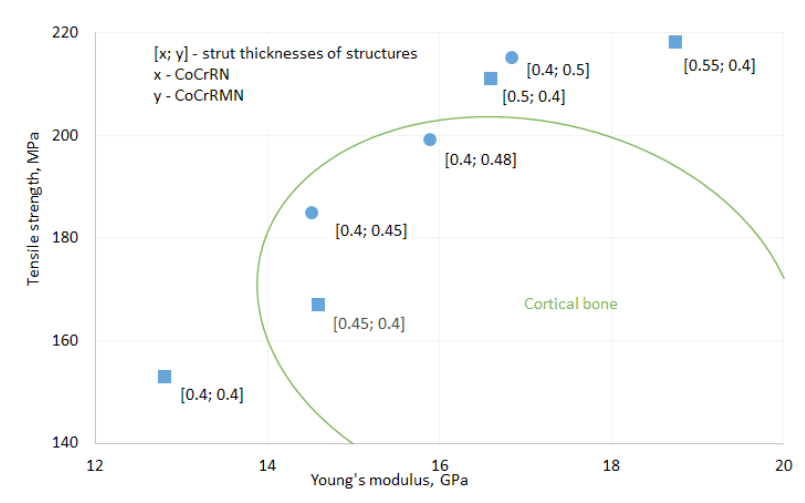

Pис. 8. (Color online) Зависимость эффективного предела прочности образцов от модуля упругости.

Fig. 8. (Color online) Dependence of the effective tensile strength of the specimens on Young's modulus.

\section{7. Заключение}

В работе проведено численное моделирование механических свойств ячеек, и их комбинаций, изготовленных методом аддитивного производства.

В результате выполненных исследований произведен отбор конфигураций ячеек по соответствию механическим и геометрическим характеристикам бедренной кости человека.

Определены конфигурации ячеек, механические свойства которых соответствуют механическим свойствам трабекулярной кости: $\mathrm{N}$ с толщиной прутка $\sim 0.48-0.56$ мм, MN с толщиной прутка $\sim 0.3-0.42$ мм, CrMN с толщиной прутка $\sim 0.3-0.35$ мм.

Получены варианты комбинаций различных конфигураций ячеек, эффективные механические свойства которых удовлетворяют механическим свойствам кортикальной кости: CoCrRMN с толщиной прутка 0.4 мм и CoCrRN с толщиной прутка 0.45 мм; CoCrRN с толщиной прутка 0.4 мм и CoCrRMN с толщиной прутка 0.45 мм; CoCrRN с толщиной прутка 0.4 мм и CoCrRMN с толщиной прутка 0.48 мм.

Использование полученных данных позволит перейти к созданию модели протеза бедренной кости человека, эффективные механические свойства которого будут соответствовать механическим свойствам кости, что позволит избежать её разрушения.

Дополнительный материал/Supplementary Material. Электронная версия статьи содержит дополнительныи материал, доступньй безвозмездно на сайте журнала (lettersonmaterials.com). / The online version of this paper 
contains supplementary material available free of charge at the journal's Web site (lettersonmaterials.com).

Благодарности /Acknowledgements. Работа выполнена в рамках реализации федеральной целевой программы «Исследования и разработки по приоритетным направлениям развития научно-технологического комплекса России на 2014-2020 годы» уникальный идентификатор проекта RFMEFI57817X0245. / This study was carried out as part of the Federal Targeted Programme "Research and Development in Priority Areas of Development of the Russian Scientific and Technological Complex for 2014-2020", unique project identifier RFMEFI57817X0245.

\section{Литература/References}

1. S. Limmahakhun, A. Oloyed, K. Sitthiseripratip, Y. Xiao, C. Yan. Additive Manufacturing. 15, 93 (2017). Crossref

2. S. Arabnejad Khanoki, D. Pasini. Journal of Biomechanical Engineering. 134 (3), 031004 (2012). Crossref

3. A. A. Popovich, V. Sh. Sufiiarov, I. A. Polozov, E. V. Borisov, D. V. Masaylo, P.N. Vopilovskiy, A.A. Sharonov, R. M. Tikhilov, A. V. Tsybin, A. N. Kovalenko, S. S. Bilyk. Biomedical Engineering. 50 (3), 202 (2016). Crossref

4. A. V. Orlov, D. V. Masaylo, V. Sh. Sufiiarov, E. V. Borisov, I. A. Polozov, A. A. Popovic. IOP Conference Series: Earth and Environmental Science. 194 (2), 022026 (2018). Crossref

5. R. Rahmanian, N. Shayesteh Moghaddam, C. Haberland, D. Dean, M. Miller, M. Elahinia. Behavior and Mechanics of Multifunctional Materials and Composites. 9058, 905814 (2014). $\underline{\text { Crossref }}$

6. M. Helou, S. Vongbunyong, S. Kara. 26th CIPR Design Conference: Procedia CIPR. 50, 94 (2016). Crossref

7. J.D. Currey. Bones Structure and Mechanics. Princeton University Press (2002) 436 p.

8. G. L. Bilich, V.L. Nikolenko. In: Atlas anatomii cheloveka 1. (Ed. by G. A. Loginova). Fenix, Russia (2014). pp.
118-121. (in Russian) [Г.Л. Билич, В. Л. Николенко. Атлас анатомии человека 1. (Под ред. Г. А. Логинова). Феникс, Россия (2014). с. 118 -121.]

9. H. Mehboob, F. Tarlochan, A. Mehboob, S.H. Chang. Materials \& Design. 149, 101 (2018). Crossref

10. M. Long, H.J. Rack. A materials science perspective. Biomaterials. 19 (18), 1621 (1998).

11. M.E. Lynch, M. Mordasky, L. Cheng, A. To. Additive Manufacturing. 22, 462 (2018). Crossref

12. A. Popovich, V. Sufiiarov, I. Polozov, E. Borisov, D. Masaylo. International Journal of Bioprinting. 2 (2), 78 (2016). $\underline{\text { Crossref }}$

13. A. V. Orlov, V.Sh. Sufiiarov, E. V. Borisov, I. A. Polozov, D. V. Masaylo, A.A. Popovich, M.O. Chukovenkova, A. V. Soklakov, D. S. Mikhaluk. Letters on materials. 9 (1), 97 (2019). Crossref

14. A. Popovich, V. Sufiiarov, E. Borisov, I. Polozov. Key Engineering Materials. 651-653, 677 (2015). Crossref

15. V. Sh. Sufiyarov, E. V. Borisov, I. A. Polozov, D. V. Masailo. Tsvetnye Metally. 7, 68 (2018). $\underline{\text { Crossref }}$

16. ANSYS ${ }^{\circledast}$ Mechanical APDL, Release 19, Help System, Mechanical APDL Theory Guide, ANSYS, Inc.

17. F. Liu, D.Z. Zhang, P. Zhang, M. Zhao, S. Jafar. Materials (Basel). 11 (3), 374 (2018). Crossref

18. M.S. Stein, S.A. Feik, C.D. Thomas, J.G. Clement, J. D. Wark. J. Bone. Miner. Res. 14 (4), 624 (1999). Crossref

19. C. Chappard, S. Bensalah, C. Olivier, P. J. Gouttenoire, A. Marchadier, C. Benhamou, F. Peyrin. Osteoporos. Int. 24 (3), 1023 (2013). Crossref

20. S. J. Hollister, C. Y. Lin, E. Saito, C. Y. Lin, R. D. Schek, J.M. Taboas, J.M. Williams, B. Partee, C.L. Flanagan, A. Diggs, E.N. Wilke, G.H. Van Lenthe, R. Muller, T. Wirtz, S. Das, S.E. Feinberg, P.H. Krebsbach. Orthod Craniofac Res. 8 (3), 162 (2005).

21. S. A Goldstein. Biomechanics. 20 (11-12), 1055 (1987). Crossref

22. M. Dumas, P. Terriault, V. Brailovski. Mater. Des. Elsevier Ltd. 121, 383 (2017). $\underline{\text { Crossref }}$ 